\title{
LETTERS
}

doi:10.1017/S1041610207006497

\section{DIY dementia screening and online assessment tools}

When confronted with the question "Should validated dementia self-assessment and screening tools be made widely available in electronic format and on the internet?", a cascade of associated questions follows, often starting with "What if ?". What if the population targeted is not internet savvy? What if individuals cannot understand the implications of the results? What if, after finding out that one might be at risk of dementia, no adequate service or treatment is available? What if these tools are abused by unscrupulous relatives? What if a false positive or false negative result is produced?

More and more people access health information on the internet and many use this information to find better treatment, to seek answers the medical system has not been able to provide, and to take ownership of their own health. Government departments, not-for-profit organizations, interest groups and commercial companies have set up websites providing information, advice and checklists relating to dementia diagnosis and treatment. Commercial ventures now advertise products on the internet to assess cognitive decline, yet validation of these products has rarely (if ever) been conducted through the usual rigorous scientific process and subjected to peer review. Consequently, the question is not so much whether individuals and their families have access to tools to screen for dementia but whether these tools have been adequately designed and validated and, more particularly, validated in the context in which they are to be used.

Our review in this issue shows that screening tests for dementia can be chosen from a long list of psychometric instruments but few have been designed or validated for self-administration, even fewer are amenable to an easy translation to the digital media, and none has been validated in digital form to be used on a computer or on the internet. The validation of a dementia screening instrument takes substantial resources, expertise and time. Therefore, it seems important to design and/or start validating such tools for digital usage (particularly on the internet) as soon as possible so that, if appropriate, they can be made widely available.

Self-assessment also presents risks. Individuals found to be at risk of dementia (and false positives) might experience dramatic decreases in selfesteem, increased depression and anxiety, and social stigma, while individuals wrongly classified as cognitively healthy might not seek the advice of specialists and delay their access to health services and treatment. A high rate of false positives could also excessively burden the health system and delay treatment for those who genuinely require it.

We are unlikely to be able to answer these questions categorically in the near future. Does it mean that we should opt for the status quo? Or should we progress cautiously with adequate mechanisms that will help us assess the value of DIY cognitive assessment? We have no simple answer to offer. Our intention is to 
stimulate an important debate and to ensure that these questions are not the exclusive domain of clinicians and academics but involve people with dementia and their families.

\section{Nicolas Cherbuin and KaARin J. Anstey}

Centre for Mental Health Research, Australian National University, Canberra, Australia

Email: nicolas.cherbuin@anu.edu.au

doi:10.1017/S1041610207006436

\section{Addenbrooke's Cognitive Examination as a better discriminator of cognitive impairment than the Mini-mental State Examination in patients with dementia}

We recently completed an audit on cognitive testing used in routine practice within our Older People Community Mental Health Team in East Dorset, U.K. We measured the frequency of use of the Addenbrooke's Cognitive Examination (ACE) following its introduction as the baseline test for cognition, comparing it to the Mini-mental State Examination (MMSE) (Folstein et al., 1975).

Published research has shown that the ACE is a better discriminator of dementia than the MMSE, offering greater sensitivity (82\%) and equal specificity (96\%) for dementia at a cut-off of 83, compared to the MMSE at a cut-off of 24 which has a sensitivity of $52 \%$ and specificity of $96 \%$ at this score (Mathuranath et al. (2000).

One of the most interesting and important findings on completion of the audit was that there was an increase in the percentage of cases that showed a disparity of scores (i.e. of significant cognitive impairment when the ACE was compared to the MMSE) between 2005 (17\%) and 2006 (36\%) when the audit was repeated. That is to say, these cases would have had a "normal" MMSE (a score of 24 or greater), but an abnormal ACE score (a score of 83 or less).

More importantly, these cases could have slipped through the screening assessment for dementia on the basis of their MMSE score alone if no other deficit of functioning was found.

Although the ACE does take a little longer than the MMSE to administer, which may affect compliance rates with this tool, we feel that this is offset by obtaining greater detail in a variety of cognitive domains, such as executive functioning, anterograde memory, episodic memory, speech and language deficits, which the MMSE does not do. We feel justified in using the ACE as a primary tool for cognitive assessments.

\section{References}

Folstein, M., Folstein, S. and McHugh, P. R. (1975). "Mini-mental state": a practical method for grading the cognitive state for the clinician. Fournal of Psychiatric Research, 12, 189-198. 